3 GOOD REASONS

To buy an HIV ring

1 It will raise awareness and empathy for the global AIDS problem, hopes its designer, Andriy Kurovets.

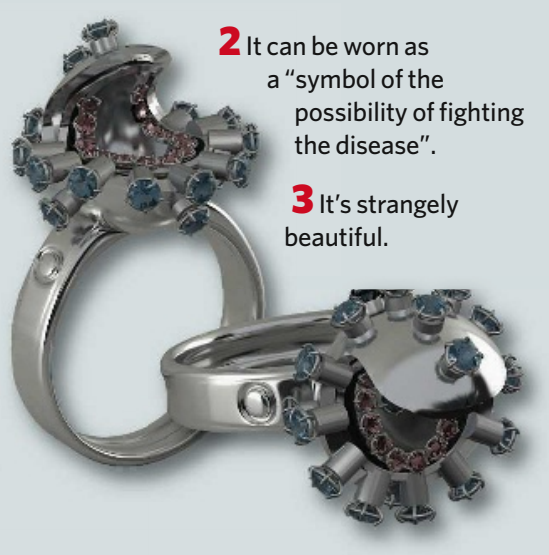

ROBOT NEWS

High ambitions

The US Defense Advanced

Research Projects Agency

(DARPA) has handed out millions of dollars for companies to design a plane that can stay aloft for five years unattended.

Low expectations

The Bristol Robotics Laboratory, UK, has won a 11 -million (US\$2 million) grant to see whether a robot can stir soup safely. The lab will test a robot's abilities to work alongside a person in an enclosed space, such as a kitchen.

\section{NUMBER CRUNCH}

E1.9 billion ( $\$ 3.8$ billion) is the estimated worth of unpaid peer review done worldwide, according to a UK report.

$\mathbf{8 . 7} \%$ of that is donated by UK researchers, despite the fact that only ...

$6.6 \%$ of journal articles are written in Britain.

\section{ON THE RECORD}

Sabine Grünwald of the Mühlenau retirement home in Hamburg, Germany, ponders how to improve the fake bus stop they installed outside the facility last year. Patients with dementia who wander off tend to collect at the stop, where they can be collected by staff. A bus never actually comes.

\title{
Near-perfect 'black'
}

Researchers working with metamaterials say that they are near to achieving a surface that can absorb every photon that hits it.

Physicist Willie Padilla's group at Boston College in Massachusetts and the team's colleagues at Duke University in Durham, North Carolina, have created structures that provide a proof of principle of these perfectly absorbing surfaces, although they have yet to stop all photons from escaping ${ }^{1}$. The researchers predict that the material will have initial applications in ultra-sensitive imaging technologies that use terahertz radiation, with wavelengths intermediate between infrared and microwaves. Terahertz imaging is a hotly tipped technology for use in medicine - fully absorbing materials at these wavelengths could help to reveal even the dimmest of features.

"Terahertz frequencies are notoriously difficult to work with - difficult to generate, and difficult to detect," says Marin Soljačić of the Massachusetts Institute of Technology in Cambridge, who is also working on controlling absorption in tailor-made metamaterials ${ }^{2}$. But there are many exciting applications that could be enabled if we mastered this technology. This work is a nice step in that direction."

Metamaterials are structures made from arrays of 'designer atoms', each of which is itself a device or structure with specially tailored properties. So far, the building blocks of most metamaterials have been designed to interact with electromagnetic radiation in unusual ways, giving the assembled metamaterial an optical behaviour that ordinary materials could never display. For example, it might have a negative refractive index, bending refracted light the 'wrong way'. Metamaterials have already provided prototypes of 'superlenses' that are more powerful than conventional ones, and 'invisibility shields' that bend electromagnetic waves smoothly around objects hidden inside.

The basic 'atoms' of these structures are C-shaped split rings made from patterned metal films on circuit boards. Radiation with a wavelength comparable to the size of the rings will stir up resonant electric currents in the metal, causing the radiation to be absorbed. For metamaterials operating at microwave frequencies, the components are relatively easy to make and are large enough to be visible.

Padilla's team has developed a new design for their perfect absorber, consisting of two back-to-back split rings. They calculated that an array of these elements should absorb about $99 \%$ of incident radiation at the resonant microwave frequency of about 11.5 gigahertz. Their experimental version managed to soak up only about $96 \%$.

But there are plenty of ways to improve this, Padilla says. One of the likely reasons for the imperfect absorbance is small errors in the shapes of the split-rings or in the accuracy of putting them together - so, more careful fabrication should produce better performance. And the researchers have now come up with an improved design that, in theory, offers $99.99 \%$ absorbance. If more than one layer of the metamaterial is used, the absorbance should be more or less perfect, they say.

Near-perfect light absorbers are needed for detectors called bolometers, which sense photons by converting their energy to heat. Bolometers made from silicon are standard items in infrared sensing, but they need to be cryogenically cooled and only work in a limited range of wavelengths. The new metamaterial absorbers work at room temperature, and the wavelength response can be tuned simply by varying the size and shape of the components.

Such an absorber would still be perfect only within a certain range of wavelengths. But this range could be broadened, to make the equivalent of a 'perfectly black' surface that absorbs fully over the entire visible spectrum by mixing different-sized split-ring components in the array. Alternatively, a perfect absorber could pick out specific 'colours', which would be useful for combining imaging and spectroscopy. "At terahertz frequencies, you could image airport luggage directly through the bag, just as with X-rays," says Padilla. "But being able to image at different wavelengths means you might obtain spectroscopic information about what you are imaging, so that you can determine if it is harmful."

Padilla and his colleagues have already managed to scale down their design to work at terahert $z$ and far-infrared frequencies ${ }^{3}$. But he says that making a perfect black in the visible range will be hard, because the components would have to be so small. A super-black material made from carbon nanotubes was reported recently ${ }^{4}$, in which the high absorbance comes from the roughness of the surface rather than the antenna action of metamaterials.

Philip Ball

Landy, N. I., Sajuyigbe, S., Mock, J. J., Smith, D. R. \& Padilla, W. J. Phys. Rev. Lett. 100, 207402 (2008)

2. Chan, D. L. C., Celanovic, I., Joannopoulos, J. D. \& Soljačić,

M. Phys. Rev. A 74, 064901 (2006).

3. Tao, H. et al. Opt. Lett. 16, 7181-7188 (2008).

4. Yang, Z.-P., Ci, L., Bur, J. A., Lin, S.-Y. \& Ajayan, P. M. Nano Lett. 8, 446-451 (2008). 